\title{
Overexpression of the orphan receptor Nur77 alters glucose metabolism in rat muscle cells and rat muscle in vivo
}

\author{
T. Kanzleiter • E. Preston • D. Wilks • B. Ho • \\ A. Benrick • J. Reznick • L. K. Heilbronn • N. Turner • \\ G. J. Cooney
}

Received: 7 September 2009 /Accepted: 29 January 2010 /Published online: 9 March 2010

(C) Springer-Verlag 2010

\begin{abstract}
Aims/hypothesis A hallmark feature of the metabolic syndrome is abnormal glucose metabolism which can be improved by exercise. Recently the orphan nuclear receptor subfamily 4, group A, member 1 (NUR77) was found to be induced by exercise in muscle and was linked to transcriptional control of genes involved in lipid and glucose metabolism. Here we investigated if overexpression of Nur77 (also known as Nr4al) in skeletal muscle has functional consequences for lipid and/or glucose metabolism. Methods L6 rat skeletal muscle myotubes were infected with a Nur77-coding adenovirus and lipid and glucose oxidation was measured. Nur77 was also overexpressed in skeletal muscle of chow- and fat-fed rats and the effects on glucose and lipid metabolism evaluated.

Results Nur77 overexpression had no effect on lipid oxidation in L6 cells or rat muscle, but did increase glucose oxidation and glycogen synthesis in L6 cells. In chow- and high-fat-fed rats, Nur77 overexpression by electrotransfer significantly increased basal glucose uptake and glycogen synthesis, but no increase in insulin-stimulated glucose metabolism was observed. Nur77 electrotransfer was associated with increased production of GLUT4 and glycogenin and increased hexokinase and phosphofructo-
\end{abstract}

T. Kanzleiter · E. Preston • D. Wilks $\cdot$ B. Ho $\cdot$ A. Benrick

J. Reznick $\cdot$ L. K. Heilbronn $\cdot$ N. Turner $\cdot$ G. J. Cooney $(\bowtie)$

Diabetes and Obesity Research Program,

Garvan Institute of Medical Research,

384 Victoria Street, Darlinghurst,

Sydney NSW 2010, Australia

e-mail: g.cooney@garvan.org.au

N. Turner $\cdot$ G. J. Cooney

St Vincent's Hospital Clinical School,

University of New South Wales,

Sydney, NSW, Australia kinase activity. Interestingly, Nur77 expression in muscle biopsies from obese men was significantly lower than in those from lean men and was closely correlated with bodyfat content and insulin sensitivity.

Conclusions/interpretation Our data provide compelling evidence that NUR77 is a functional regulator of glucose metabolism in skeletal muscle in vivo. Importantly, the diminished content in muscle of obese insulin-resistant men suggests that it might be a potential therapeutic target for the treatment of dysregulated glucose metabolism.

Keywords Electrotransfer - Glucose metabolism . Insulin resistance $\cdot$ Nur77 · Obesity · Skeletal muscle

$\begin{array}{ll}\text { Abbreviations } \\ \text { EDL } & \text { Extensor digitorum longus } \\ \text { FFM } & \text { Fat-free mass } \\ \text { GFP } & \text { Green fluorescent protein } \\ \text { GIR } & \text { Glucose-infusion rate } \\ \text { NR4A } & \text { Nuclear receptor subfamily 4, group A } \\ \text { NUR77 } & \text { Nuclear receptor subfamily 4, group A, } \\ & \text { member 1 } \\ \text { TA } & \text { Tibialis anterior }\end{array}$

\section{Introduction}

In the last two decades there has been a dramatic increase in the prevalence of obesity, such that it now represents one of the most significant health concerns worldwide [1]. Obesity can give rise to a multitude of pathological conditions commonly referred to as the metabolic syndrome. One possible outcome of this rapid increase in obesity-related diseases is that children born early in the 21 st century will 
have a lower life expectancy than their parents [2]. One of the most common diseases related to obesity is type 2 diabetes, the prevalence of which is also increasing at an alarming rate [3]. The key metabolic defect predisposing to the development of type 2 diabetes is insulin resistance, which is the relative failure of insulin to exert its multiple biological effects on carbohydrate and lipid metabolism [4]. Insulin resistance is also an underlying defect common to several other components of the metabolic syndrome, including obesity, hypertension, dyslipidaemias and cardiovascular disease. Therefore, unravelling the complex molecular mechanisms responsible for the development of insulin resistance represents a major priority in the fight against the rising prevalence of type 2 diabetes and other metabolic disorders.

Skeletal muscle represents approximately $40 \%$ of body mass and accounts for $70-80 \%$ of postprandial insulinstimulated glucose uptake $[5,6]$. Defects in insulinstimulated glucose uptake and glycogen synthesis in muscle have been suggested to be important early events in the pathogenesis of insulin resistance [4]. It is also well established that regular physical exercise improves insulin action in skeletal muscle [7, 8]. The beneficial effects of exercise include acute events regulating signalling pathways to increase glucose metabolism, as well as chronic adaptations of skeletal muscle involving coordinated expression of glucose metabolic genes [9, 10].

Recently, it was reported that the expression of Nur77 (also known as Nr4al) was induced in human and rodent skeletal muscle following exercise [11, 12]. We have also shown in skeletal muscle cells that the expression of Nur 77 can be induced by two exerciseactivated pathways, beta-adrenergic stimulation and calcium influx [13]. Furthermore, Nur77 expression is reduced in animal models of obesity and insulin resistance, where responsiveness to beta-adrenergic stimulation is diminished $[13,14]$. Nuclear receptor subfamily 4 , group A, member 1 (NUR77) has been shown to be a transcriptional regulator of genes linked to glucose as well as lipid metabolism in muscle $[15,16]$; however, little is known regarding the functional consequences of altered NUR77 production in skeletal muscle on glucose and/or lipid metabolism. The aim of this study was to therefore investigate the effect of Nur77 overexpression on lipid and glucose metabolism in skeletal muscle.

\section{Methods}

Cell culture L6 rat skeletal muscle myoblasts were cultured in six well plates and maintained in alpha-MEM (Invitrogen, Carlsbad, CA, USA) supplemented with antibiotics and 10\% (vol./vol.) FCS (Sigma-Aldrich, St Louis, MO, USA). At
$70-80 \%$ confluence the medium was changed to alphaMEM supplemented with $2 \%$ (vol./vol.) FCS to initiate differentiation. At 7 days post-differentiation cells were incubated for $24 \mathrm{~h}$ with adenoviruses $\left(5 \times 10^{6}\right.$ plaqueforming units $[\mathrm{PFU}] / \mathrm{ml}$ ) coding for NUR77 or green fluorescent protein (GFP) as control (generously provided by S. Tetradis, UCLA School of Dentistry, Los Angeles, CA, USA) and used for experiments $48 \mathrm{~h}$ after infection.

Animal study Male Wistar rats weighing approximately $250 \mathrm{~g}$ were purchased from the Animal Resources Centre (Perth, WA, Australia). The animals were kept in a temperature-controlled room $\left(22 \pm 1^{\circ} \mathrm{C}\right)$ on a $12 \mathrm{~h} \mathrm{light/dark}$ cycle with free access to food and water. Rats were fed ad libitum for a period of 3 weeks with a standard laboratory diet or a high-fat diet as previously described [17]. All experiments were carried out with the approval of the Garvan Institute/St Vincent's Hospital Animal Experimentation Ethics Committee, following guidelines issued by the National Health and Medical Research Council of Australia.

In vivo electroporation and euglycaemic-hyperinsulinaemic clamp studies with glucose tracers were performed as described previously $[18,19]$. For the in vivo electroporation experiments, Nur77 was expressed in the right tibialis anterior (TA) and extensor digitorum longus (EDL) muscle and empty vector (pCDNA3.1, Invitrogen) was expressed in the left leg as an internal control. One week before the tracer studies, the jugular vein and carotid artery of rats designated to undergo clamp studies, or the jugular vein alone for rats designated to undergo basal tracer uptake studies, were cannulated. Rats were singly housed and handled daily for the following week to minimise stress. Body weight was recorded daily, and only rats that had fully recovered to their pre-surgery weight were subsequently studied. Animals were food restricted overnight (65\% of normal intake) before the tracer experiments were performed. At the end of each study, rats were killed by intravenous injection of pentobarbitone sodium (Nembutal; Abbott Laboratories, Sydney, NSW, Australia) and muscles rapidly dissected and freeze-clamped using liquid nitrogencooled tongs. Plasma tracer disappearance curves and the counts of phosphorylated 2-deoxy- $\left[{ }^{3} \mathrm{H}\right]$ glucose and $\left[{ }^{14} \mathrm{C}\right]$ glucose in glycogen extracts from individual muscles were used to calculate tissue-specific glucose uptake and glycogen synthesis, respectively [20].

Human study Sedentary, non-smoking, non-diabetic obese and lean men (age 21-49 years) were recruited. The study protocol was approved by the Human Research Ethics Committee at St Vincent's Hospital, Sydney, and participants provided informed written consent. Participants were tested after a $10 \mathrm{~h}$ overnight fast. Weight was measured in light clothing and height was assessed by a stadiometer. Following 
this, a vastus lateralis muscle biopsy was performed and the samples were blotted for blood and any visible fat was removed before they were snap frozen at $-80^{\circ} \mathrm{C}$. A standard 120 min hyperinsulinaemic-euglycaemic clamp was then performed with indirect calorimetry (Deltatrac; Datex, Helsinki, Finland) according to previously described studies [21]. Immediately following the clamp body fat was measured by dual-energy X-ray absorptiometry (Lunar DPX-Lunar Radiation, Madison, WI, USA).

$R N A$ isolation and quantitative real-time PCR For cells and tissue samples RNA was isolated according to the manufacturer's instructions using TRI-reagent (Sigma-Aldrich). RNA integrity was evaluated on agarose gels and concentration was determined spectrophotometrically using a Nanodrop spectrophotometer (Biolab, Scoresby, VIC, Australia). RNA was converted into cDNA using random primers (New England Biolabs, Ipswich, MA, USA) and the cDNA synthesis kit from Qiagen (Mississauga, ON, USA). Taqman assays for Nur77 (Rn00577766_m1, Hs00374230_m1; Applied Biosystems, Foster City, CA, USA) and Universal Probe Library assays (Roche Diagnostics, Indianapolis, IN, USA) for glycogenin (5'-tcttgtgg cttctgtagaaagga-3'; 5'gaggatacaaacggetgtgc-3') and Glut4 (also known as Slc2a4) (5'-ctgtgccatcctgatgactg-3'; 5'-cgtagctcatggctggaact-3') were employed to quantify mRNA expression. PCR runs were performed on an ABI 9600HT cycler (Applied Biosystems, Foster City, CA, USA) or Roche LC480 (Roche Diagnostics, Indianapolis, IN, USA). A standard curve, consisting of serial dilutions of pooled cDNA samples, was measured with every PCR run to quantify the respective transcripts. Expression data for Nur77, glycogenin and Glut4 were normalised to 18S rRNA expression.

Measurement of fatty acid and glucose oxidation For fatty acid oxidation, L6 cells transfected with adenovirus coding for NUR77 or GFP were incubated with alpha-MEM medium containing $0.5 \mathrm{mmol} / 1$ oleate (Sigma-Aldrich) coupled to $2 \%$ BSA (wt/vol.; Sigma-Aldrich) and $\left[1-{ }^{14} \mathrm{C}\right]$ oleate (GE Healthcare, Rydalmere, NSW, Australia) at a final concentration of $37 \mathrm{kBq} / \mathrm{ml}$. After $1 \mathrm{~h}$ of incubation at $37^{\circ} \mathrm{C}$ in a sealed plate the medium was transferred to a sealed glass vial, acidified with $1 \mathrm{~mol} / \mathrm{l}$ perchloric acid. Liberated ${ }^{14} \mathrm{CO}_{2}$ was captured in $1 \mathrm{~mol} / \mathrm{l} \mathrm{NaOH}$ and measured by liquid scintillation counting. To determine the ${ }^{14} \mathrm{C}$ counts present in the acid-soluble metabolites we performed a 'Folch extraction' of the cells, measured the radioactivity in the aqueous phase and combined these values with the ${ }^{14} \mathrm{CO}_{2}$ values to give the total fatty acid oxidation rate. Fatty acid oxidation in EDL strips was conducted as described in detail elsewhere [22].

Glucose oxidation and glycogen synthesis rates in Nur77- and Gfp-transfected L6 cells were assessed at $37^{\circ} \mathrm{C}$ by incubating cells for $1 \mathrm{~h}$ in alpha-MEM containing $37 \mathrm{kBq} / \mathrm{ml}\left[\mathrm{U}_{-}{ }^{14} \mathrm{C}\right]$ glucose (GE Healthcare) and, where indicated, $100 \mathrm{nmol} / 1$ insulin. At the completion of the incubation the medium was transferred to a sealed glass vial and acidified with $1 \mathrm{~mol} / 1$ perchloric acid. Liberated ${ }^{14} \mathrm{CO}_{2}$ was captured in $1 \mathrm{~mol} / 1 \mathrm{NaOH}$ and measured by liquid scintillation counting. Glycogen was extracted from the cells after dissolving tissue with $1 \mathrm{~mol} / 1 \mathrm{KOH}$ and precipitating glycogen with $95 \%$ (vol./vol.) ethanol. $\left[{ }^{14} \mathrm{C}\right]$ Glucose incorporation into precipitated glycogen was measured by liquid scintillation counting. Furthermore, the total glycogen amount was determined after digestion with amyloglucosidase and colorimetric determination of glucose content.

Immunoblot analysis Frozen muscle tissue was powdered and solubilised in radioimmunoprecipitation assay (RIPA) buffer supplemented with complete protease inhibitor cocktail and PhosStop phosphatase inhibitor (Roche Diagnostics, Indianapolis, IN, USA). Protein concentration was determined using the method of Bradford (BioRad Laboratories, Gladesville, NSW, Australia). Protein, $50 \mu \mathrm{g}$, was diluted in Laemmli SDS buffer and separated on an SDS gel. After transfer to a polyvinylidene fluoride (PVDF) membrane, antibodies against GLUT1, GLUT4 (both generously provided by D. James, Garvan Institute, Sydney, NSW, Australia), glycogen synthase (kindly provided by J. Lawrence, University of Virginia, Charlottesville, VA, USA) and glycogenin (Santa Cruz Biotechnology, Santa Cruz, CA, USA) were used for the immunodetection of respective proteins.

Enzyme assays Measurements of hexokinase and phosphofructokinase activity in homogenates of electroporated tibialis muscles were performed as described in detail elsewhere [23].

Statistical analysis Values are given as mean \pm SEM. Significance was tested for by Student's $t$ test if comparisons between two groups were made or two-way ANOVA followed by the Holm-Sidak post hoc test if multiple treatments were compared. Where appropriate, paired $t$ tests or ANOVA on repeated measures were used. Calculations were performed with SigmaStat 3.5 or Graphpad Prism 5, and $p$ values less than 0.05 were considered to be significant.

\section{Results}

Effect of Nur77 on lipid and glucose metabolism Adenoviral overexpression of Nur77 in L6 myotubes and electroporation of Nur77 into rat skeletal muscle were used to assess the effects of this nuclear hormone receptor on substrate metabolism. In L6 myotubes Nur77 mRNA expression was increased on average 80-fold compared 
with control infected cells $(1,482 \pm 117$ vs $19 \pm 3$ arbitrary units) while in rat skeletal muscle Nur 77 mRNA expression was elevated approximately 30 -fold in the test leg compared with the control leg $(17,112 \pm 2,373$ vs $561 \pm 64$ arbitrary units). Fatty acid oxidation was assessed and Nur77 overexpression had no effect on this variable in either the L6 myotubes (Fig. 1a) or rat EDL muscle strips (Fig. 1b). In contrast, Nur77 overexpression increased glucose oxidation $(+30 \%)$ in L6 myotubes in both the absence and presence of insulin (100 nmol/l) (Fig. 2a). As a second endpoint of glucose metabolism, $\left[{ }^{14} \mathrm{C}\right]$ glucose incorporation into glycogen was also measured and L6 myotubes overexpressing Nur77 displayed significantly higher rates of glucose incorporation into glycogen compared with control infected myotubes (Fig. 2b). Since glycogen is a strong regulator of its own synthesis we also measured total glycogen content but could not detect any differences between L6-Nur77 and control myotubes (data not shown).

Effect of NUR77 on glucose uptake and glucose incorporation into glycogen in vivo Our initial in vitro experiments suggested that NUR77 influences glucose, but not lipid, metabolism and therefore we sought to investigate the effect of NUR77 on glucose metabolism in vivo. Nur77 was overexpressed in rat skeletal muscle by electroporation and we examined glucose uptake and glucose incorporation into glycogen under basal and insulin-stimulated conditions. Basal glucose uptake was significantly increased $(+70 \%)$ in TA muscle overexpressing Nur77 compared with contralateral control muscles (Fig. 3a). Glucose incorporation into glycogen was also increased $(+120 \%)$ in TA muscle overexpressing Nur 77 as compared with the control muscle (Fig. 3b). Both of the above variables were also measured in red quadriceps muscle of the left and right leg and no significant differences were observed (data not

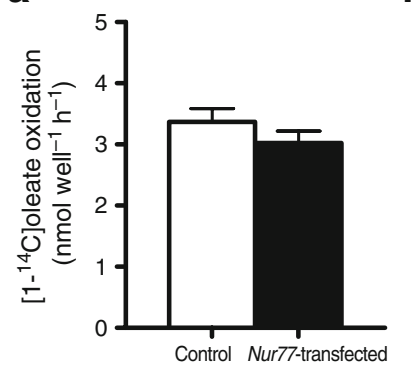

L6 myotubes

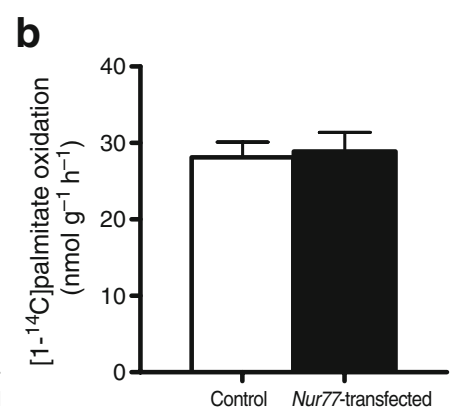

Rat EDL muscle
Fig. 1 Lipid oxidation in a L6 myotubes and b rat skeletal muscle. L6 myotubes were infected with NUR77-coding adenovirus (black bars) or GFP control virus (white bars) and the oxidation of $\left[1-{ }^{14} \mathrm{C}\right]$ oleate was measured for $1 \mathrm{~h}$ at $37^{\circ} \mathrm{C}$. Rat EDL muscle was transfected with Nur77 (black bars) or empty vector (white bars) and the oxidation of $\left[1-{ }^{14} \mathrm{C}\right]$ palmitate was measured for $1 \mathrm{~h}$ at $30^{\circ} \mathrm{C}$. Data are presented as mean \pm SEM of $n=4$ L6 myotubes and $n=12$ rat EDL muscle samples
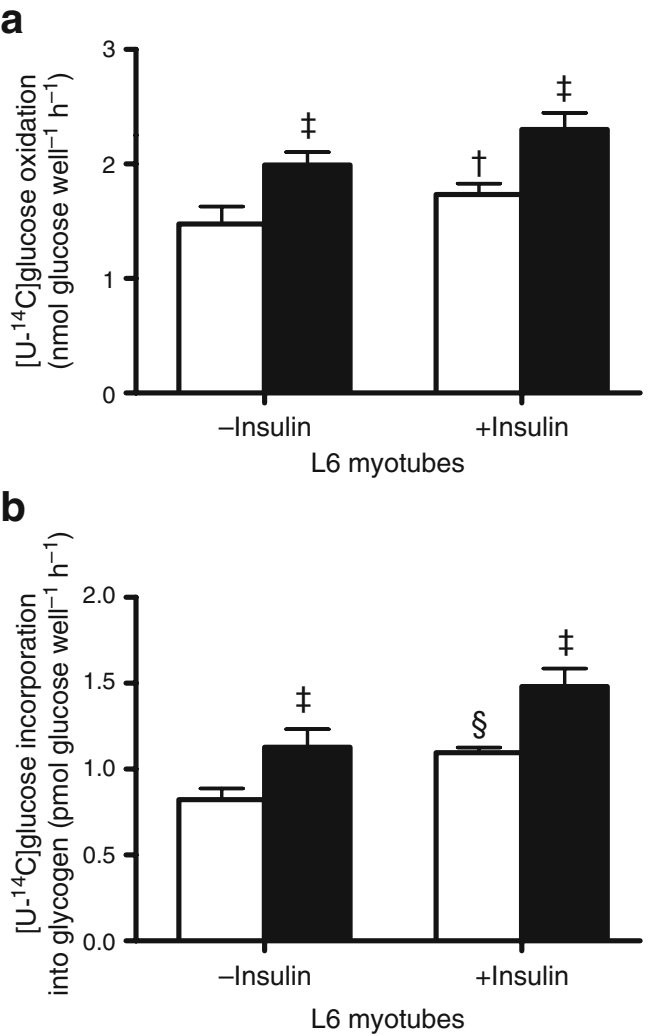

Fig. 2 Assessment of glucose metabolism in L6 myotubes. L6 myotubes were infected with Nur77-coding adenovirus (black bars) or GFP control virus (white bars) and the oxidation of $\left[\mathrm{U}-{ }^{14} \mathrm{C}\right]$ glucose (a) or its incorporation into glycogen (b) was measured for $1 \mathrm{~h}$ at $37^{\circ} \mathrm{C}$ in the absence or presence of $100 \mathrm{nmol} / 1$ insulin. Two-way ANOVA followed by Holm-Sidak pairwise comparison revealed a significant effect of Nur77 and insulin in both experiments. Data are presented as mean \pm SEM of $n=9$ independent experiments. ${ }^{\dagger} p=0.03$ vs - insulin, ${ }^{\ddagger} p=0.0002$ vs - insulin, ${ }^{\S} p=0.0006$ vs - insulin

shown), thereby confirming that the changes in glucose metabolism were specific to the TA muscle overexpressing Nur77.

Considering the impressive changes observed under basal conditions, we next measured the effect of Nur 77 overexpression on glucose metabolism in muscle under insulin-stimulated euglycaemic conditions ( $\sim 5 \mathrm{mmol} / \mathrm{l}$ blood glucose and $4 \mathrm{mU} \mathrm{kg}^{-1} \mathrm{~min}^{-1}$ insulin infusion). Insulinstimulated glucose uptake was slightly elevated in muscle overexpressing Nur77 compared with the control muscle, but this failed to reach statistical significance (Fig. 3a). Glucose incorporation into glycogen was also not different between Nur77-overexpressing and control muscles following insulin stimulation (Fig 3b).

Consequences of Nur77 overexpression on glucose metabolism in insulin-resistant rats In order to assess if Nur77 overexpression could augment glucose metabolism in muscle of insulin-resistant rats, we fed rats a high-fat diet for 3 weeks. This diet resulted in a significant increase in 
a

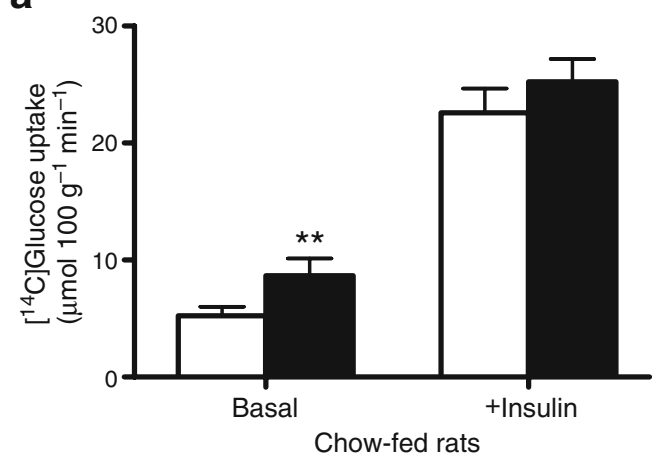

b

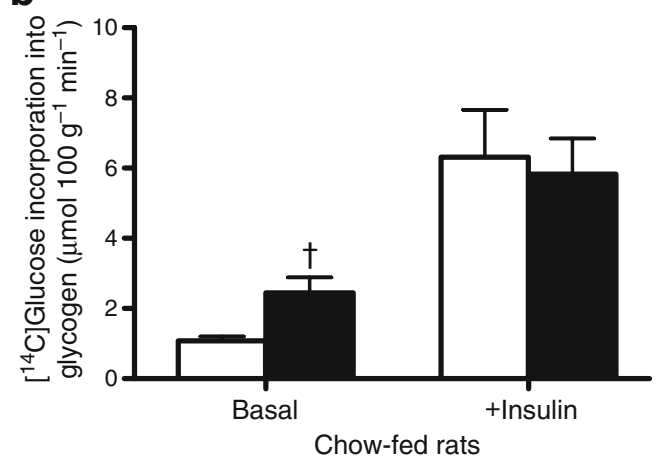

Fig. 3 Effect of NUR77 on muscle glucose uptake and glycogen synthesis in chow-fed rats. Seven days after in vivo electroporation of the TA muscle with Nur77 (black bars) or empty vector (white bars), glucose tracer studies with 2-deoxy-D-[2,6- $\left.{ }^{3} \mathrm{H}_{2}\right]$ glucose and $\left[\mathrm{U}-{ }^{14} \mathrm{C}\right]$ glucose were performed. Tissue-specific glucose uptake (a) and $\left[{ }^{14} \mathrm{C}\right]$ glucose incorporation into glycogen (b) were assessed under basal and hyperinsulinaemic-euglycaemic conditions. Data are presented as mean \pm SEM of $n=8-9$ rats. ${ }^{* *} p=0.01,{ }^{\dagger} p=0.007$ as determined by paired Student's $t$ test

body weight (Table 1) as compared with chow-fed animals undergoing the same procedure. Insulin action was assessed with a hyperinsulinaemic-euglycaemic clamp and the glucose infusion rate during the clamp was significantly reduced, confirming insulin resistance in these animals (Table 1). Similar to our findings in chow-fed animals, we observed a significant increase in glucose uptake and incorporation of glucose into glycogen in Nur77-overexpressing muscle in the basal state (Fig. 4a, b). In the insulin-stimulated fat-fed animals, glucose uptake and incorporation into glycogen were significantly lower than in the corresponding chow-fed animals (Fig. 3a, b), but we found no difference between Nur77-overexpressing and control muscles (Fig. 4a, b).

Downstream targets of NUR77 In order to investigate the mechanisms that might underpin the observed increase in glucose uptake under basal conditions, we examined known and putative downstream targets of NUR77. Glut4 expression has been reported to be regulated by NUR77 [16] and analysis of several promoter regions of genes related to glucose metabolism revealed the sequence of putative NUR77 response elements in the promoter of glycogen synthase and glycogenin.

We found the protein content of GLUT4 to be significantly upregulated $(40-80 \%)$ in the TA muscles overexpressing Nur77 compared with controls in both chow- and fat-fed animals (Fig. 5a, b). The self-glucosylating primer of glycogen synthesis, glycogenin, was significantly upregulated in the Nur77-overexpressing tibialis of chow-fed animals; however, in the high-fat-fed animals there was no observable increase in glycogenin content in response to Nur77 overexpression (Fig. 5a, b). Despite the putative NUR77-binding site in its promoter region, the protein levels of glycogen synthase were not changed in response to Nur77 overexpression (Fig. 5a, b). To exclude upregulation of the other major glucose transporter in skeletal muscle as the cause for increased glucose uptake under basal conditions with Nur77 overexpression, we also measured the protein expression of GLUT1. No differences were observed in GLUT1 protein between Nur77-overexpressing and control muscles under both chow-fed and high-fat-fed conditions (Fig. 5a, b).

Enzyme activity Another possible cause for increased muscle glucose uptake could be a change in the activity

Table 1 Physiological characteristics of rats undergoing glucose tracer studies

\begin{tabular}{|c|c|c|c|c|}
\hline \multirow[t]{2}{*}{ Variable } & \multicolumn{2}{|l|}{ Chow-fed rats } & \multicolumn{2}{|l|}{ High-fat-fed rats } \\
\hline & Basal animals $(n=9)$ & Clamp animals $(n=8)$ & Basal animals $(n=9)$ & Clamp animals $(n=7)$ \\
\hline Body mass (g) & $284.2 \pm 5.9$ & $266.8 \pm 4.7$ & $345.0 \pm 10.9^{* * *}$ & $315.3 \pm 6.3^{* * *}$ \\
\hline Fasting blood glucose (mmol/l) & $4.51 \pm 0.1$ & $4.50 \pm 0.15$ & $4.61 \pm 0.1$ & $4.94 \pm 0.25$ \\
\hline Glucose infusion rate $\left(\mathrm{mg} \mathrm{kg} \mathrm{min}^{-1}\right)$ & NA & $31.6 \pm 1.1$ & NA & $23.1 \pm 1.94^{* * *}$ \\
\hline \multirow[t]{2}{*}{ Plasma insulin (pg/ml) } & $1,418 \pm 220$ & Pre: $950 \pm 139$ & $2,116 \pm 122^{* *}$ & Pre: $1,729 \pm 578$ \\
\hline & & Post: $4,085 \pm 420$ & & Post: $3,913 \pm 160$ \\
\hline
\end{tabular}

High-fat-fed rats vs chow-fed rats: ${ }^{* *} p<0.01 ;{ }^{* * *} p<0.001$

NA, not applicable 
a

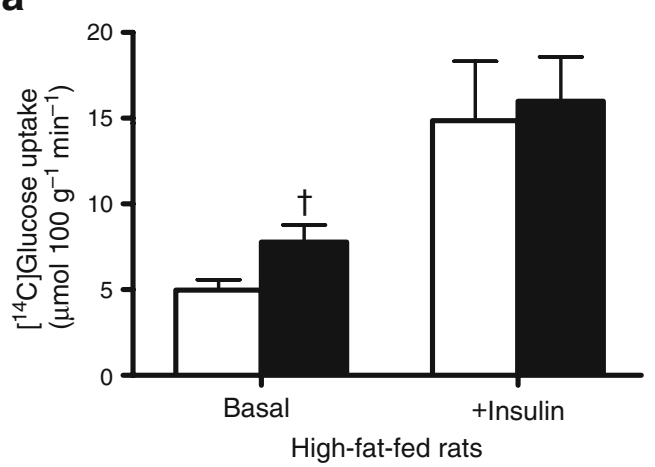

b

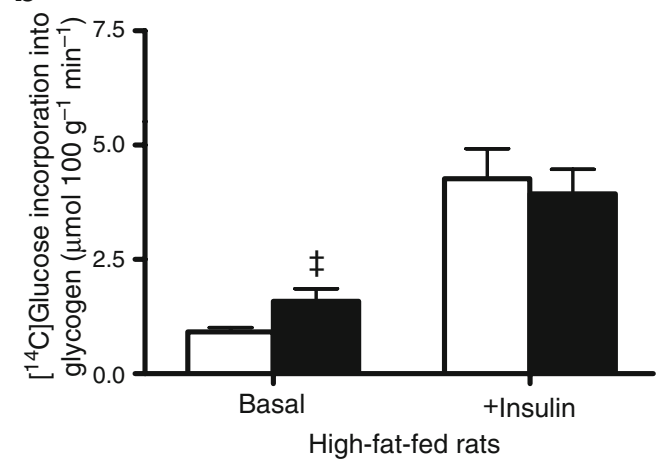

Fig. 4 Effect of NUR77 on muscle glucose uptake and glycogen synthesis in high-fat-fed rats. Rats were fed a high-fat diet for 3 weeks and TA muscles were transfected with Nur77 (black bars) or empty vector (white bars) and the effect on tissue-specific glucose uptake (a) and glucose incorporation into glycogen (b) was tested under basal conditions and during a hyperinsulinaemic-euglycaemic clamp. Data are presented as mean \pm SEM of $n=7-10$ rats. ${ }^{\dagger} p=0.005,{ }^{\star} p=0.02$ as determined by paired Student's $t$ test

of glycolytic enzymes (e.g. hexokinase and phosphofructokinase), which can be rate limiting for glucose uptake under insulin-stimulated conditions or during exercise [24, 25]. Measurement of hexokinase activity revealed a significant increase in response to Nur77 expression in chow-fed animals. Interestingly, this increase in hexokinase activity was not observed in high-fat-fed rats (Fig. 6a). In a similar fashion, the activity of phosphofructokinase was significantly increased in response to Nur77 overexpression in chow-fed rats, but this increase was blunted in high-fat-fed rats (Fig. 6b).

Nur77 expression in skeletal muscle is reduced in obese humans Recently, we reported that expression of Nur77 is reduced in the muscle of rats that had been fed a high-fat diet for 3 weeks [13]. Given the beneficial effects of NUR77 on basal glucose uptake, we also measured Nur77 expression in muscle biopsies (vastus lateralis) from obese and lean men. Obese men $(n=12)$ weighed significantly more $(100.3 \pm 1.9 \mathrm{~kg}$ vs $78.2 \pm 5.4 \mathrm{~kg} ; p<0.0005)$ and had a higher body-fat content $(33 \pm 2 \%$ fat vs $18 \pm 2 \%$ fat; $p<$
$0.00002)$ than the control group of lean individuals $(n=9)$. Additionally, the obese men had significantly higher fasting blood glucose $(5.7 \pm 0.5 \mathrm{mmol}$ vs $4.9 \pm 0.6 \mathrm{mmol} ; p<$ $0.0005)$ and serum insulin levels $(17.3 \pm 7.4 \mathrm{mU} / \mathrm{ml}$ vs $8.7 \pm 4.3 \mathrm{mU} / \mathrm{ml} ; p<0.007)$. Insulin sensitivity as measured by hyperinsulinaemic-euglycaemic glucose clamps was also significantly impaired in the obese men as compared with the lean study group (glucose-infusion rate [GIR]: $35.4 \pm 3.7 \mu \mathrm{mol}$ fat-free mass $[\mathrm{FFM}]^{-1} \mathrm{~min}^{-1}$ vs $84.9 \pm$ $\left.9.4 \mu \mathrm{mol} \mathrm{FFM}{ }^{-1} \min ^{-1} ; p<0.00003\right)$. In line with our previous studies in rodents [13], Nur77 expression was significantly decreased in obese men (Fig. 7a). The expression of the putative NUR77 target, glycogenin, was also reduced in muscle of obese men compared with lean controls (Fig. 7b); however, Glut4 expression was not different between the two groups ( $1.28 \pm 0.09$ vs $1.42 \pm 0.06$ arbitrary units, obese vs lean). Interestingly, Nur77 expression was closely correlated with body-fat content (Fig. 7c), glucose infusion rate (Fig. 7d) and its putative downstream target glycogenin $\left(R^{2}=0.38, p=0.015\right)$.

\section{Discussion}

The nuclear orphan receptor NUR77 has been reported to regulate the transcription of genes linked to lipid and glucose metabolism in skeletal muscle cells $[15,16]$. This leads to speculation that this transcription factor may be important in regulating substrate metabolism in the context of insulin resistance and obesity. We recently showed [13] that two exercise-activated pathways increase Nur77 expression in a skeletal muscle cell line and other studies have reported that physical exercise in humans and rodents increases Nur77 expression in muscle [11, 12]. Therefore, NUR77 may be an important link between the transcriptional regulation of lipid and glucose metabolism and physical exercise, which is known to improve substrate metabolism in insulin-resistant animals and humans $[8,26$, 27]. A caveat to many of the previous studies is that the conclusions have been largely based on NUR77-mediated changes in gene expression. However, whether changes in NUR77 impact functional measures of glucose and lipid metabolism in vitro or in vivo has not previously been assessed.

In this study, we overexpressed Nur77 in L6 myotubes and in rat skeletal muscle and measured the effect on lipid and glucose oxidation in vitro. The magnitude of increase in Nur77 expression achieved is relatively similar to that reported following adrenergic stimulation of both muscle cells and skeletal muscle in vivo [13, 16, 28]. Nur77 overexpression did not significantly change lipid oxidation, whereas two endpoints of glucose metabolism (glucose oxidation and glycogen synthesis) were significantly 
a
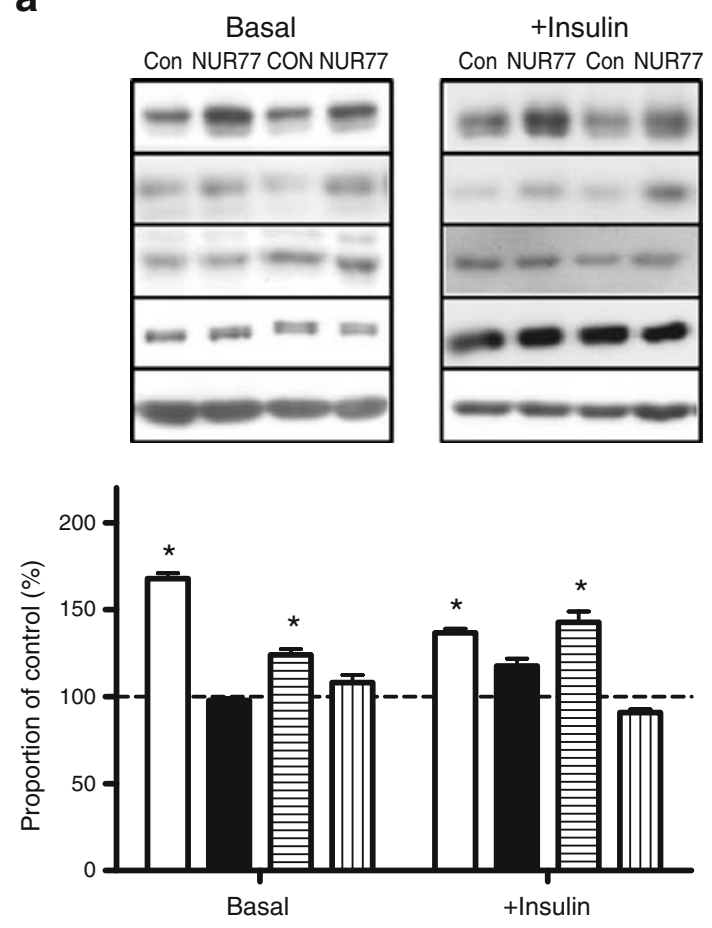

Fig. 5 Immunoblotting analyses of GLUT4 (white bars), GLUT1 (black bars), glycogenin (GYG; horizontal lines), and glycogen synthase (GYS; vertical lines) protein content in tibialis muscle of chow-fed (a) and high-fat-fed (b) rats. Left legs were electroporated with empty vectors and right legs overexpressed Nur77. Densitometric

elevated. These findings suggest that NUR77 is a functional regulator of glucose metabolism in muscle, but is not involved in the regulation of fatty acid oxidation. These results might initially seem contradictory to the findings of Maxwell et al. [16], who used $\mathrm{C} 2 \mathrm{C} 12$ cells with a stable knockdown of Nur77 and identified differentially expressed genes linked to lipid and glucose metabolism. However, the loss of Nur77 expression in Nur77-knockout mice results in a compensatory increase of another member of nuclear receptor subfamily 4, group A (NR4A), Nor1 (also known as $N r 4 a 3)$ [15, 29]. This partial redundancy between NR4A receptors makes it difficult to study the direct effects of gene deletion. Therefore, a rise in Norl expression in response to NUR77 knockdown could potentially explain the increase in genes linked to lipid metabolism. Indeed, a recent study showed that NOR1 regulates palmitate oxidation in skeletal muscle cells [28]. Further evidence against a direct role for NUR77 in the regulation of fatty acid metabolism comes from a study by Chao and colleagues [15] who overexpressed Nur77 in $\mathrm{C} 2 \mathrm{C} 12$ cells and did not observe any differential expression of genes linked to fatty acid metabolism, but did see alterations in genes linked to glucose metabolism. These apparent differences in NUR77 function highlight the fact that data
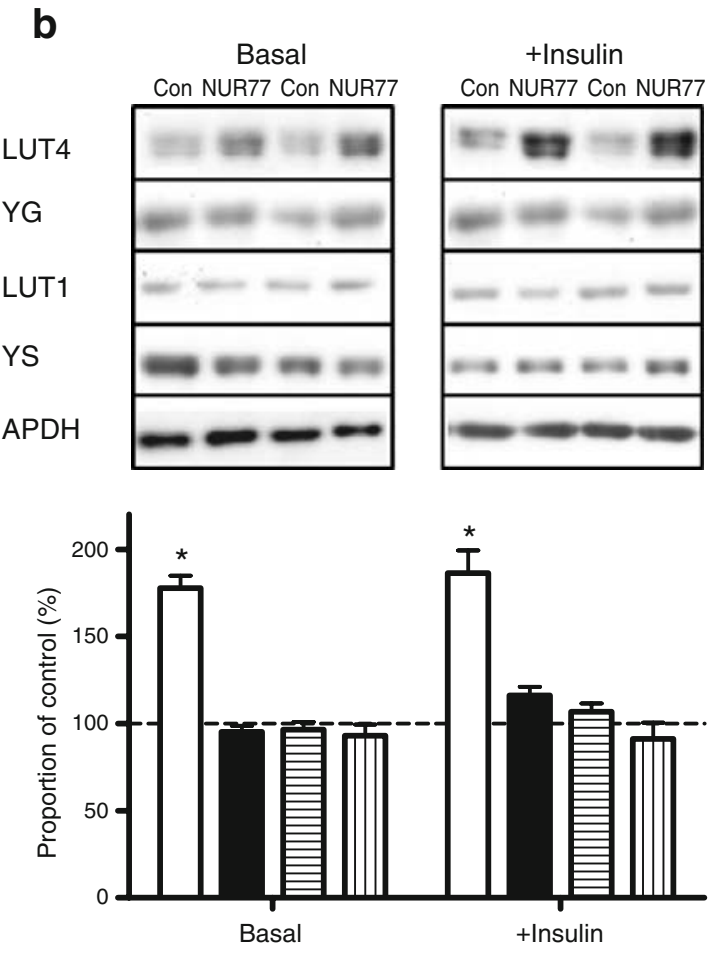

values were normalised to the left control leg and expressed as the percentage increase. Data are presented as mean \pm SEM of at least six rats; differences were considered to be significant for ${ }^{*} p<0.05$ as determined by paired Student's $t$ test. Con, control; GAPDH, glyceraldehyde-3-phosphate dehydrogenase

obtained from overexpression is not necessarily the opposite of knockdown.

To assess the functional role of NUR77 on glucose metabolism in vivo, we measured glucose uptake and glycogen synthesis under basal and insulin-stimulated conditions in control and transfected TA muscle in rats. Consistent with our findings in vitro, we found that basal glucose uptake and incorporation into glycogen was increased in muscle overexpressing Nur77 and this was associated with an increase in GLUT4 and glycogenin content, as well as elevated activity of the glycolytic enzymes, hexokinase and phosphofructokinase. Interestingly, under insulin-stimulated conditions, we observed only a very minor effect of Nur77 overexpression on glucose uptake and glycogen synthesis, despite the fact that GLUT4 content was increased. These findings of significant NUR77 effects on glucose metabolism in the basal but not insulinstimulated state were observed in both chow- and fat-fed animals. There are several reasons why Nur77 overexpression might result in significant effects on glucose metabolism in the basal but not the insulin-stimulated state even though GLUT4 protein was increased in both situations.

First, it might be difficult to observe an increase in glucose uptake in insulin-stimulated muscle of a similar magnitude to 
a

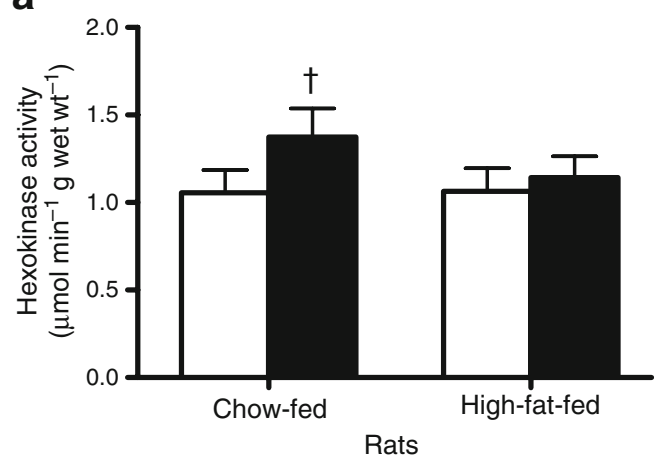

b

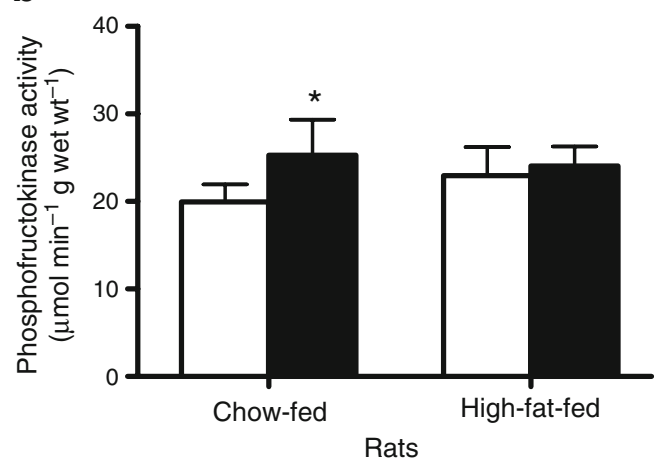

Fig. 6 Activity of glycolytic enzymes was measured in muscle electroporated with Nur77 (black bars) or empty vector (white bars). a Hexokinase activity of $n=14$ chow-fed and $n=11$ high-fat-fed rats ( $p=0.03$ ). b Phosphofructokinase activity of $n=12$ chow-fed and $n=$ 13 high-fat-fed rats $(* p=0.05)$

that observed in muscle in the basal state because such an increase (approximately $4 \mu \mathrm{mol} 100 \mathrm{~g}^{-1} \mathrm{~min}^{-1}$ ) falls within the margins of variation normally observed in determining glucose metabolism in muscle from hyperinsulinaemiceuglycaemic clamped rats $[18,19]$.

Second, the transport of glucose across the membrane by GLUT4 might not be the rate-limiting step under insulinstimulated conditions. There is increasing evidence that under basal conditions the transport of glucose across the membrane is the rate-limiting step in muscle glucose uptake but under insulin-stimulated conditions or during exercise the membrane has increased permeability to glucose and this step is no longer rate limiting [24, 25, 30-34]. Under these conditions delivery of glucose via the bloodstream, phosphorylation inside the cells as well as usage further down the glycolytic pathway become increasingly more important. This is also evident in Glut4 transgenic mice where the amount of increased GLUT4 content (fourfold) does not directly translate to an increase in muscle glucose uptake of a similar magnitude (1.9-fold) [35, 36].

The improvement in glucose incorporation into glycogen under basal conditions could also be the result of increased GLUT4 content and increased availability of intracellular glucose. However, putative NUR77 response elements have been revealed by sequence analysis in the promoter regions of genes encoding other important proteins in glycogen synthesis. Glycogenin protein content was increased in response to Nur77 overexpression in chow-fed animals, suggesting that it is indeed a NUR77 downstream target. Both glycogenin and Glut4 expression are consistently upregulated in muscle after exercise in humans [37], and exercise is also known to increase Nur77 expression [11, 12]. The activity of hexokinase, another exercise-induced gene $[9,10]$, was also significantly increased in response to NUR77 overproduction. Collectively these findings suggest that NUR77 may mediate some of the beneficial effects of exercise on glucose metabolism. The attenuation of NUR77-induced changes in several of the above proteins in high-fat-fed rats might have contributed to the smaller increase in basal glucose uptake observed. Why high-fat feeding blunted the increase in glycogenin content as well as hexokinase and phosphofructokinase activity in response to Nur77 overexpression is not clear, but this may represent another manifestation of the effects of a high-fat diet on muscle glucose metabolism.

We also observed significantly reduced expression of NUR77 in the muscles of insulin-resistant obese men, as well as a lower expression of glycogenin. NUR77 expression was also closely correlated with body-fat content and a

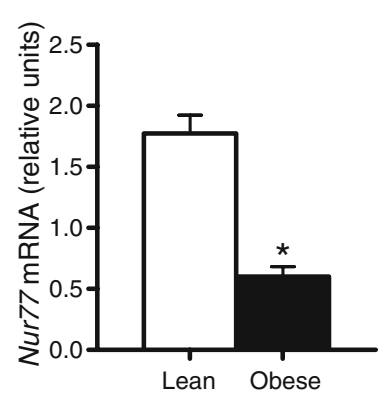

C

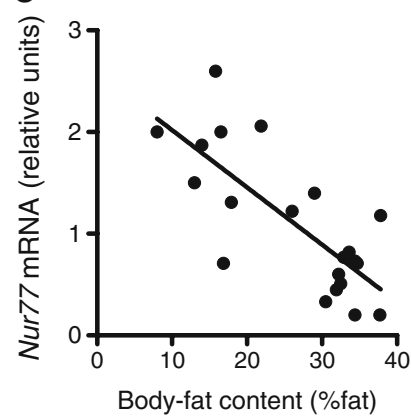

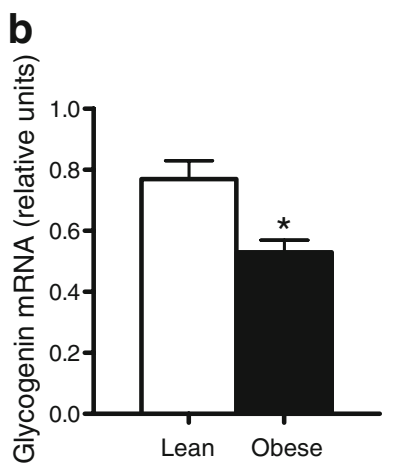

d

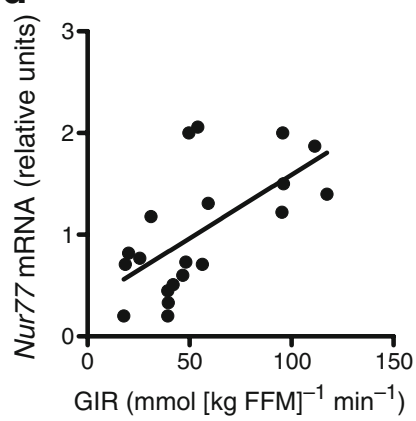

Fig. 7 Nur77 (a) and glycogenin (b) mRNA expression in vastus lateralis muscle of lean $(n=4-11)$ and obese $(n=12)$ men. Values are presented as mean \pm SEM, $* p<0.05$. Correlation of Nur77 with $\mathbf{c}$ body-fat content $\left(R^{2}=0.58, p=0.0001\right)$ and $\mathbf{d}$ glucose-infusion rate $\left(R^{2}=0.40, p=0.002\right)$ in study participants 
insulin sensitivity, suggesting a possible functional role for NUR77 in glucose metabolism in humans. Our current findings, and those from a recent report showing increased NUR77 expression in human muscles following exercise [12], suggest that NUR77 may be a useful target for treating disorders of glucose metabolism.

The data presented here demonstrate that acute overexpression of the nuclear hormone transcription factor Nur77 in skeletal muscle can increase the capacity for glucose uptake and glycogen synthesis. In a physiological context this might be of relevance in the post-exercise state where Nur77 as well as Glut4 and glycogenin expression are increased to presumably meet the demand for replenishing energy stores [37, 38]. Increased expression of Nur77 after exercise $[11,12]$ and a decrease in insulin-resistant rats [13] and humans (Fig. 7) suggest that this regulator of glucose metabolism might be an interesting target in the context of increasing glucose metabolism in muscle. Although overexpression of Nur77 did not improve insulin-stimulated glucose metabolism after high-fat-diet feeding in rats, the augmentation in the basal state would suggest an overall benefit to glucose metabolism by increasing NUR77 activity in insulin-resistant muscle.

In summary, we provide evidence for a functional role of the orphan nuclear receptor NUR77 in glucose metabolism in skeletal muscle. The beneficial effects on glucose metabolism are associated with alterations in GLUT4, glycogenin, hexokinase and phosphofructokinase. These new data support previous studies showing that NUR77 is induced after exercise bouts in skeletal muscle and is reduced in obesity and suggest that manipulation of NUR77 could provide beneficial effects on muscle glucose metabolism.

Acknowledgements This study was supported by Deutsche Forschungsgemeinschaft (DFG), Diabetes Australia Research Trust and the National Health and Medical Research Council (NHMRC) of Australia (Project grant 276419). T. Kanzleiter is supported by a DFG Research Fellowship, J. Reznick is supported by an Australian Postgraduate Award, N. Turner is supported by a Career Development Award and G. J. Cooney by a Research Fellowship from the NHMRC of Australia. The authors are grateful to G. Frangioudakis and F. Hochgraefe for their critical comments on this manuscript.

Duality of interest The authors declare that there is no duality of interest associated with this manuscript.

\section{References}

1. Kelly T, Yang W, Chen CS, Reynolds K, He J (2008) Global burden of obesity in 2005 and projections to 2030. Int J Obes (Lond) 32:1431-1437

2. Olshansky SJ, Passaro DJ, Hershow RC et al (2005) A potential decline in life expectancy in the United States in the 21 st century. N Engl J Med 352:1138-1145
3. Wild S, Roglic G, Green A, Sicree R, King H (2004) Global prevalence of diabetes: estimates for the year 2000 and projections for 2030. Diabetes Care 27:1047-1053

4. Petersen KF, Shulman GI (2006) Etiology of insulin resistance. Am J Med 119:S10-S16

5. DeFronzo RA, Jacot E, Jequier E, Maeder E, Wahren J, Felber JP (1981) The effect of insulin on the disposal of intravenous glucose. Results from indirect calorimetry and hepatic and femoral venous catheterization. Diabetes 30:1000-1007

6. Nuutila P, Knuuti MJ, Raitakari M et al (1994) Effect of antilipolysis on heart and skeletal muscle glucose uptake in overnight fasted humans. Am J Physiol 267:E941-E946

7. Shojaee-Moradie F, Baynes KC, Pentecost C et al (2007) Exercise training reduces fatty acid availability and improves the insulin sensitivity of glucose metabolism. Diabetologia 50:404-413

8. Knowler WC, Barrett-Connor E, Fowler SE et al (2002) Reduction in the incidence of type 2 diabetes with lifestyle intervention or metformin. N Engl J Med 346:393-403

9. Frosig C, Rose AJ, Treebak JT, Kiens B, Richter EA, Wojtaszewski JF (2007) Effects of endurance exercise training on insulin signaling in human skeletal muscle: interactions at the level of phosphatidylinositol 3-kinase, Akt, and AS160. Diabetes 56: 2093-2102

10. Hildebrandt AL, Pilegaard H, Neufer PD (2003) Differential transcriptional activation of select metabolic genes in response to variations in exercise intensity and duration. Am J Physiol Endocrinol Metab 285:E1021-E1027

11. Kawasaki E, Hokari F, Sasaki M, Sakai A, Koshinaka K, Kawanaka K (2009) Role of local muscle contractile activity in the exercise-induced increase in NR4A receptor mRNA expression. J Appl Physiol 106:1826-1831

12. Mahoney DJ, Parise G, Melov S, Safdar A, Tarnopolsky MA (2005) Analysis of global mRNA expression in human skeletal muscle during recovery from endurance exercise. FASEB $\mathrm{J}$ 19:1498-1500

13. Kanzleiter T, Wilks D, Preston E, Ye J, Frangioudakis G, Cooney GJ (2009) Regulation of the nuclear hormone receptor nur77 in muscle: influence of exercise-activated pathways in vitro and obesity in vivo. Biochim Biophys Acta 1792:777-782

14. Lessard SJ, Rivas DA, Chen ZP et al (2009) Impaired skeletal muscle $\beta$-adrenergic activation and lipolysis are associated with whole-body insulin resistance in rats bred for low intrinsic exercise capacity. Endocrinology 150:883-891

15. Chao LC, Zhang Z, Pei L, Saito T, Tontonoz P, Pilch PF (2007) Nur77 coordinately regulates expression of genes linked to glucose metabolism in skeletal muscle. Mol Endocrinol 21:2152-2163

16. Maxwell MA, Cleasby ME, Harding A, Stark A, Cooney GJ, Muscat GEO (2005) Nur77 regulates lipolysis in skeletal muscle cells - evidence for cross-talk between the beta-adrenergic and an orphan nuclear hormone receptor pathway. J Biol Chem 280: $12573-12584$

17. Turner N, Bruce CR, Beale SM et al (2007) Excess lipid availability increases mitochondrial fatty acid oxidative capacity in muscle: evidence against a role for reduced fatty acid oxidation in lipidinduced insulin resistance in rodents. Diabetes 56:2085-2092

18. Cleasby ME, Davey JR, Reinten TA et al (2005) Acute bidirectional manipulation of muscle glucose uptake by in vivo electrotransfer of constructs targeting glucose transporter genes. Diabetes 54:2702-2711

19. Bruce CR, Hoy AJ, Turner N et al (2009) Overexpression of carnitine palmitoyltransferase-1 in skeletal muscle is sufficient to enhance fatty acid oxidation and improve high-fat diet-induced insulin resistance. Diabetes 58:550-558

20. Kraegen EW, James DE, Jenkins AB, Chisholm DJ (1985) Doseresponse curves for in vivo insulin sensitivity in individual tissues in rats. Am J Physiol 248:E353-E362 
21. Heilbronn LK, Gan SK, Turner N, Campbell LV, Chisholm DJ (2007) Markers of mitochondrial biogenesis and metabolism are lower in overweight and obese insulin-resistant subjects. J Clin Endocrinol Metab 92:1467-1473

22. Bruce CR, Brolin C, Turner N et al (2007) Overexpression of carnitine palmitoyltransferase $\mathrm{I}$ in skeletal muscle in vivo increases fatty acid oxidation and reduces triacylglycerol esterification. Am J Physiol Endocrinol Metab 292:E1231-E1237

23. MacArthur DG, Seto JT, Chan S et al (2008) An Actn3 knockout mouse provides mechanistic insights into the association between alpha-actinin-3 deficiency and human athletic performance. Hum Mol Genet 17:1076-1086

24. Fueger PT, Bracy DP, Malabanan CM, Pencek RR, Granner DK, Wasserman DH (2004) Hexokinase II overexpression improves exercise-stimulated but not insulin-stimulated muscle glucose uptake in high-fat-fed C57BL/6J mice. Diabetes 53:306-314

25. Petersen HA, Fueger PT, Bracy DP, Wasserman DH, Halseth AE (2003) Fiber type-specific determinants of Vmax for insulinstimulated muscle glucose uptake in vivo. Am J Physiol Endocrinol Metab 284:E541-E548

26. Holloszy JO (1967) Biochemical adaptations in muscle. Effects of exercise on mitochondrial oxygen uptake and respiratory enzyme activity in skeletal muscle. J Biol Chem 242:2278-2282

27. Tuomilehto J, Lindstrom J, Eriksson JG et al (2001) Prevention of type 2 diabetes mellitus by changes in lifestyle among subjects with impaired glucose tolerance. N Engl J Med 344:1343-1350

28. Pearen MA, Myers SA, Raichur S, Ryall JG, Lynch GS, Muscat GE (2008) The orphan nuclear receptor, NOR-1, a target of betaadrenergic signaling, regulates gene expression that controls oxidative metabolism in skeletal muscle. Endocrinology 149:2853-2865

29. Kanzleiter T, Schneider T, Walter I et al (2005) Evidence for $\mathrm{Nr} 4 \mathrm{a} 1$ as a cold-induced effector of brown fat thermogenesis. Physiol Genomics 24:37-44
30. Darragh J, Soloaga A, Beardmore VA et al (2005) MSKs are required for the transcription of the nuclear orphan receptors Nur77, Nurr1 and Nor1 downstream of MAPK signalling. Biochem J 390:749-759

31. Fueger PT, Heikkinen S, Bracy DP et al (2003) Hexokinase II partial knockout impairs exercise-stimulated glucose uptake in oxidative muscles of mice. Am J Physiol Endocrinol Metab 285: E958-E963

32. Halseth AE, Bracy DP, Wasserman DH (1998) Limitations to exercise- and maximal insulin-stimulated muscle glucose uptake. J Appl Physiol 85:2305-2313

33. Halseth AE, Bracy DP, Wasserman DH (2000) Limitations to basal and insulin-stimulated skeletal muscle glucose uptake in the high-fat-fed rat. Am J Physiol Endocrinol Metab 279:E1064 E1071

34. Halseth AE, Bracy DP, Wasserman DH (2001) Functional limitations to glucose uptake in muscles comprised of different fiber types. Am J Physiol Endocrinol Metab 280:E994-E999

35. Hansen PA, Gulve EA, Marshall BA et al (1995) Skeletal muscle glucose transport and metabolism are enhanced in transgenic mice overexpressing the Glut4 glucose transporter. J Biol Chem 270:1679-1684

36. Ren JM, Marshall BA, Mueckler MM, McCaleb M, Amatruda JM, Shulman GI (1995) Overexpression of Glut4 protein in muscle increases basal and insulin-stimulated whole body glucose disposal in conscious mice. J Clin Invest 95:429-432

37. Kraniou Y, Cameron-Smith D, Misso M, Collier G, Hargreaves M (2000) Effects of exercise on GLUT-4 and glycogenin gene expression in human skeletal muscle. J Appl Physiol 88:794 796

38. Kuo CH, Browning KS, Ivy JL (1999) Regulation of GLUT4 protein expression and glycogen storage after prolonged exercise. Acta Physiol Scand 165:193-201 